\title{
Optical properties of electrochemically etched N-type silicon wafers for solar cell applications
}

\author{
Martin Králik ${ }^{1}$, Matej Goraus², Emil Pinčík ${ }^{3}$
}

\begin{abstract}
The presented experiments and studies are intended for photovoltaic applications of crystalline silicon. This work deals with chemical treatment of the surface of n-type silicon wafers with different resistivity to reduce their reflectivity. Chemical surface treatment of silicon is an alternative method to using the antireflection layer. Optical losses caused by the reflection of light from the surface of the solar cells significantly reduce their efficiency. The investigated samples were prepared by the electrochemical etching method in the solution based on hydrofluoric acid and ethanol. The analysis of the prepared samples is divided into two parts, namely experimental measurements, and theoretical modeling. Experimental measurements are performed using UV-VIS spectroscopy, spectroscopic ellipsometry and SEM microscopy. Theoretical modeling is based on the construction and optimization of theoretical model of optical response (reflectivity and ellipsometric parameters) to determine the effective refractive index and thickness of formed structure. Effective refractive index of studied samples in theoretical model of optical response is based on Looyenga effective medium approximation and Tauc-Lorentz dispersion model.
\end{abstract}

K e y w ords: electrochemical etching, effective medium approximation, Looyenga EMA, modified Fresnel coefficients, reflectivity, Tauc-Lorentz dispersion model

\section{Introduction}

The optical losses due to high surface reflectivity of solar cells based on silicon are currently the important issue that needs to be resolved in photovoltaic science. The use of antireflection coating (ARC) is currently a most widely used method for reducing reflectivity of solar cells. The reflectivity reduction of most commercial silicon solar cells is based on single layer $\mathrm{SiO}_{2}, \mathrm{~S}_{3} \mathrm{~N}_{4}$ and $\mathrm{TiO}_{2}$ ARC [1]. The results published by G. Hashmi et al compare the influence of different ARC on the silicon reflectivity, from the above studies, the most significant reduction in reflectivity is observed for $\mathrm{Si}_{3} \mathrm{~N}_{4}$ ARC with a thickness of $\sim 74 \mathrm{~nm}$. The silicon reflectivity using $\mathrm{Si}_{3} \mathrm{~N}_{4}$ ARC before surface passivation is below $10 \%$ in the wavelength range from $475(2.61 \mathrm{eV})$ to $\sim 875 \mathrm{~nm}(1.42 \mathrm{eV})[2]$.

However, the use of ARC also has disadvantages, such as reduction of reflectivity in a relatively narrow spectrum of wavelengths and their relatively complicated preparation. This work deals with the possibilities of effective reduction of reflectivity in a wide range of wavelengths using chemical surface treatment of crystalline silicon wafers. By chemical surface treatment of silicon, it is possible to achieve a reflectivity of below $1.5 \%$. The silicon surface with such low reflectivity appears as black, from this reason it is usually called black silicon. Due to their low spectral reflectivity, no additional application of antireflective layers is necessary. However, the low reflectiv- ity does not necessarily mean high conversion efficiency. Increased surface recombination due to the larger surface area of the nanostructures has a significant negative influence on conversion efficiency. The problem of surface recombination was effectively resolved in the work of H. Savin et al [3].

There are several technological processes of chemical surface treatment of silicon, while the most commonly used technological processes currently are electrochemical etching, stain etching, metal-assisted chemical etching, reactive ion etching, surface structure chemical transfer (SSCT), anisotropic wet etching, etc [4-8]. The result of these technological processes are different structures (porous structure, pyramidal structure, nanocrystalline structure) with different properties.

Our main aim is to effectively reduce the surface reflectivity of silicon structures using a low-cost, simple, and effective chemical method. The above requirements for the chemical method can be met by using the electrochemical etching method. In this work we present optical properties of samples prepared by electrochemical etching method. The result of the electrochemical etching method is in most cases a porous structure, which currently belongs to the materials with a wide range of industrial applications (sensors, as anodes in Lithium-ion battery, photovoltaic applications, biosensors, drug delivery, optoelectronics, self-cleaning coatings, etc) [9-20].

\footnotetext{
${ }^{1}$ Institute of Aurel Stodola, Faculty of Electrical Engineering and Information Technology, University of Žilina, ul. kpt. J. Nálepku 1390, 03101 Liptovský Mikuláš, Slovakia, martin.kralik@fel.uniza.sk, ${ }^{2}$ Physics Department, Faculty of Electrical Engineering and Information Technology, University of Žilina, Univerzitná 1, 01026 Žilina, Slovakia, ${ }^{3}$ Institute of Physics, Slovak Academy of Sciences, Dúbravská cesta 9, 84511 Bratislava, Slovakia
}

DOI:10.2478/jee-2020-0055, Print (till 2015) ISSN 1335-3632, On-line ISSN 1339-309X

(C) This is an open access article licensed under the Creative Commons Attribution-NonCommercial-NoDerivs License (http: //creativecommons.org/licenses/by-nc-nd/3.0/). 


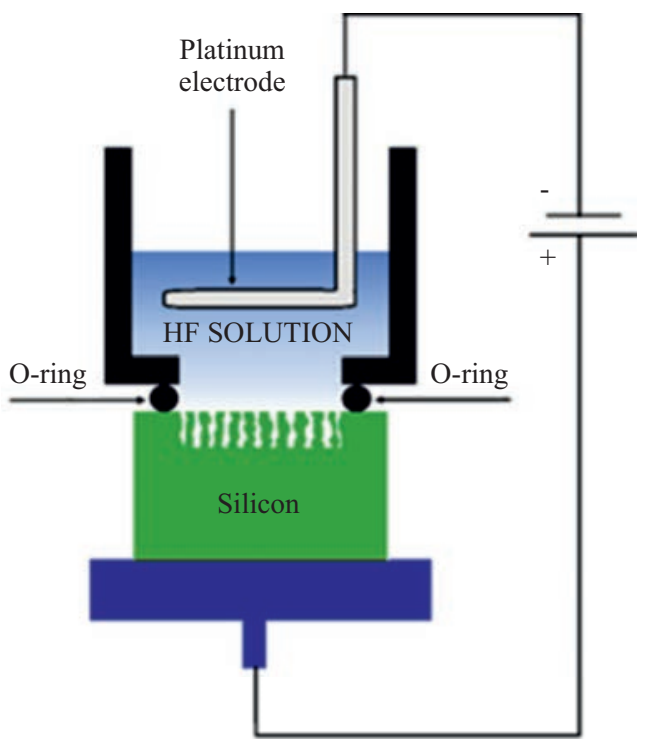

Fig. 1. Basic schematic of electrochemical etching method

It is important to note that homogeneous porous structures do not have a sufficiently low reflectivity and are therefore not suitable for photovoltaic applications. However, the reflectivity of inhomogeneous porous structures is low enough for these structures to be used in the field of photovoltaic applications. Electrochemical etching method is a suitable method for producing inhomogeneous porous structures, as it is possible to control the formation of inhomogeneities by changing the etching conditions (current density, electrolyte composition, etching time, etc) during the production process [4-7]. In addition to changing the etching conditions during the production process, the type and concentration of dopants in the substrate also have a significant effect on the homogeneity of the structure. The study of the influence of substrate resistivity on the homogeneity of the resulting structure and its optical properties is the main subject of this work.

\section{Experiment}

Samples published in this work were prepared by electrochemical etching of boron-doped silicon ( $\mathrm{Si}$ ) wafers (ON SEMICONDUCTORS) with different dopant concentrations and thus different resistivity. The Si wafers were etched in solution of $49 \%$ hydrofluoric acid (HF) and absolute ethanol $(1: 1)$ for 30 minutes. The chemicals used in this experiment were from CENTRALCHEM company. The current density in the etching process was $28.3 \mathrm{mAcm}^{-2}$. The basic schematic of electrochemical etching method used to production our samples is shown in Fig. 1. Silicon wafer with a resistivity of $\sim 0.06 \Omega \mathrm{cm}$ was used to produce sample $\mathrm{S} 1$ and silicon wafer with a resistivity of $\sim 1-10 \Omega \mathrm{cm}$ was used to produce sample S2. After the etching process, the samples were passivated in nitrogen atmosphere. The samples were analyzed by experimental measurement of reflectivity at normal incidence. Sample S2 was also analyzed by spectroscopic ellipsometry. An aluminum mirror was used as a reference in the reflectivity measurement.

\section{Results and discussions}

The experimental part of analysis of optical properties of prepared samples consisted of measurements of optical response using spectroscopic ellipsometry and UVVIS spectroscopy (measurements of reflectivity). The theoretical part of analysis of optical properties consisted of construction and optimization of theoretical model of optical response. As this is a fundamental research into the production of low-reflectance silicon structures for solar cell applications, more important than an ideal theoretical model of the optical response is low reflectivity of these structures. Theoretical modeling of the optical response was performed mainly to determine the thickness of the formed structure on the surface of substrate. To improve the theoretical model of optical response, SEM measurements were performed. It can be seen from the SEM micrographs (Fig. 2 and Fig. 5) that all samples show an inhomogeneous structure. For this reason, we decided to use the Looyenga effective medium approximation (LEMA) to describe the effective dielectric function in the theoretical model of the optical response. The LEMA formula does not consider a regular geometry of the incrustations and is defined by equation [21]

$$
\epsilon_{\mathrm{eff}}=(1-f) \epsilon_{\mathrm{m}}^{1 / 3}+f \epsilon_{\mathrm{p}}^{1 / 3},
$$

where $\varepsilon_{m}$ is dielectric function of matrix material, $\epsilon_{p}$ is dielectric function of particle material and $f$ is volume fraction of particle material. The dielectric function of the matrix material in LEMA was modeled using the TaucLorentz dispersion model and the dielectric function of particle material was air. The complex dielectric function expressed by the Tauc-Lorentz dispersion model is defined by the relation [22]

$$
\begin{aligned}
& \varepsilon_{\mathrm{i}}(E)=\frac{1}{E} \sum_{i=1}^{N} \frac{A_{i} E_{i} C_{i}\left(E-E_{g}\right)^{2}}{\left(E^{2}-E_{i}^{2}\right)^{2}+C_{i}^{2} E^{2}}, \\
& \varepsilon_{\mathrm{r}}(E)=\varepsilon_{\mathrm{r}}(\infty)+\frac{2}{\pi} P \int_{E_{\mathrm{g}}}^{\infty} \frac{\xi \varepsilon_{\mathrm{i}}(\xi)}{\xi^{2}-E^{2}} \mathrm{~d} \xi,
\end{aligned}
$$

where index $r$ and $i$ represents real and imaginary part of complex dielectric function, $E_{g}$ is the optical band gap energy, $A_{i}, E_{i}, C_{i}$ are the fitting parameters and $N$ is the number of oscillators equation (3) is valid only for energies greater than $E_{g}$ and is equal to zero for other energy values. This equation represents the Kramers-Kronig integral and its numerical solution is presented in the work [22]. Tauc-Lorentz dispersion model was chosen due to its consistency with Kramers-Kronig causality (3). The effective thickness and volume fraction of effective complex refractive index of sample $\mathrm{S} 1$ and $\mathrm{S} 2$ are listed in 

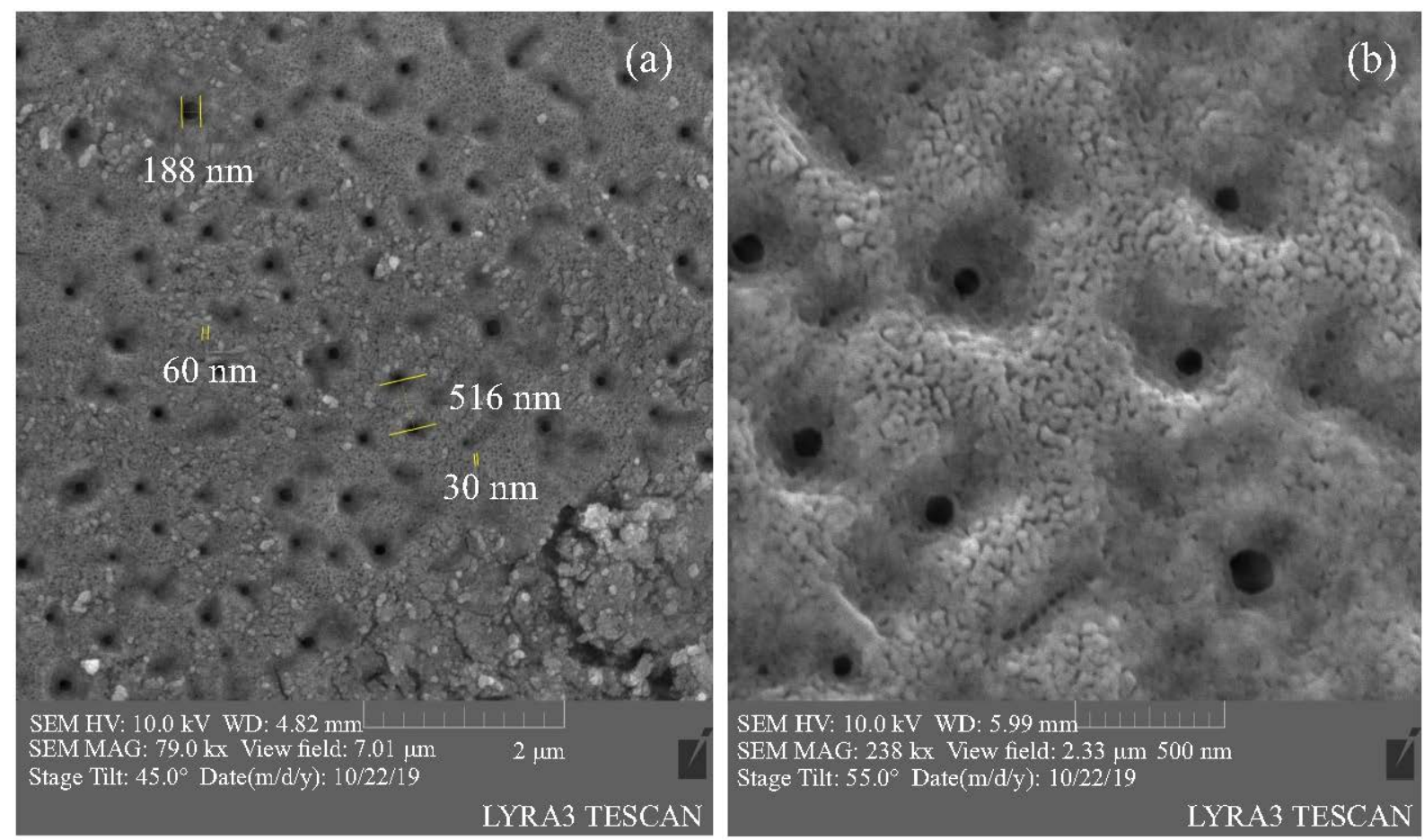

Fig. 2. Top view SEM micrograph of the sample S1 scale: (a) $-2 \mu \mathrm{m}$, and (b) $-500 \mathrm{~nm}$

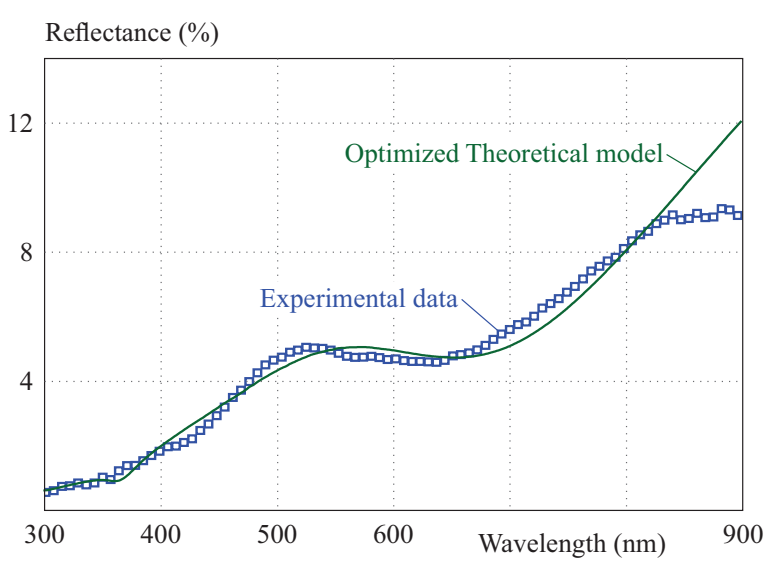

Fig. 3. Experimental data: $R$ of sample $\mathrm{S} 1$ and optimized theoretical model of sample $\mathrm{S} 1$

Table 1.

\begin{tabular}{lcc} 
Sample & $f_{\text {air }}$ & Thickness $R(\mathrm{~nm})$ \\
\hline $\mathrm{S} 1$ & 0.80 & 83 \\
$\mathrm{~S} 2$ & 0.75 & 136 \\
\hline
\end{tabular}

Tab. 1. The effective thickness calculation was performed 5 times for each sample.

Electrochemical etching of $\mathrm{Si}$ wafer with resistivity $\sim 0.06 \Omega \mathrm{cm}$ (sample S1) caused the formation of a low reflective structure on its surface. The most pronounced decrease in $R$ is observed in the wavelength range from $400 \mathrm{~nm}$ to $\sim 650 \mathrm{~nm}$ (below $\sim 5.1 \%$ ). It can be seen from the SEM micrograph (Fig. 2) that this is a relatively complicated inhomogeneous porous structure with

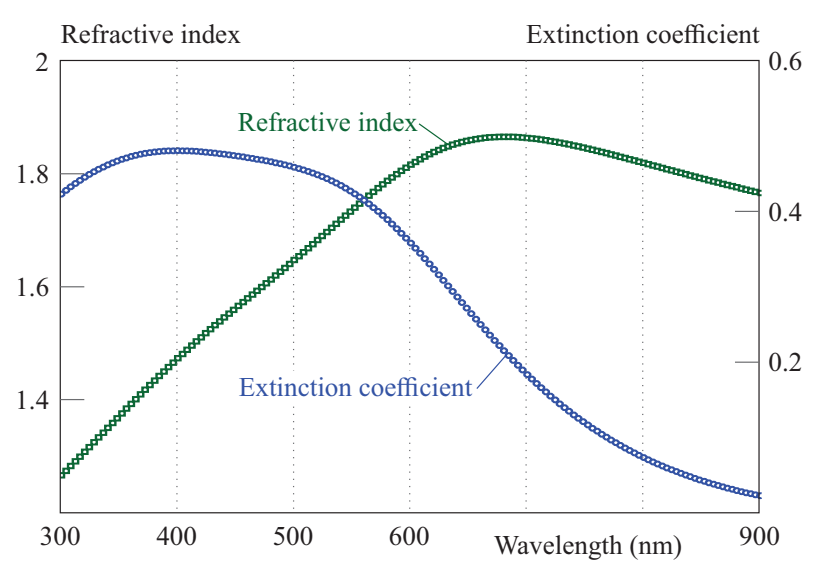

Fig. 4. Effective refractive index and extinction coefficient of sample S1

a pore diameter about $188 \mathrm{~nm}$. To determine the effective thickness of the formed structure, we created and optimized a theoretical model of $R$. Theoretical model of $R$ was based on Looyenga effective medium approximation and Tauc-Lorentz dispersion model. The optimized parameters of Tauc-Lorentz dispersion formula are listed in Tab. 2. Figure 3 shows the experimental data of $R$ of sample $\mathrm{S} 1$ and their optimized theoretical model. The effective refractive index and extinction coefficient of sample S1 obtained from optimized theoretical model of optical response are shown in Fig. 4. The optimization of the experimental data obtained from the ellipsometric measurements was not performed due to high noisy experimental data, probably due to the high roughness and inhomogeneity of the sample surface. 

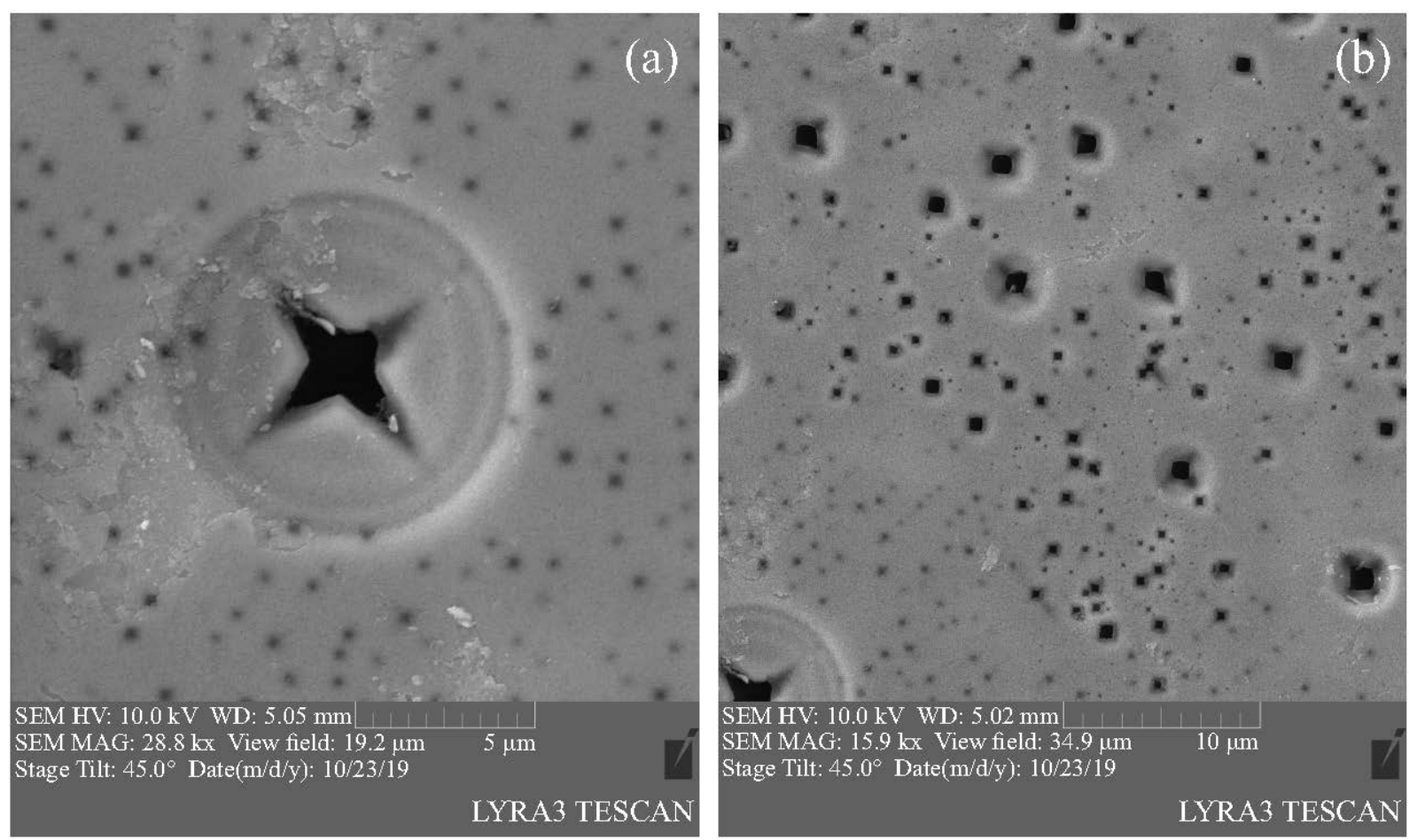

Fig. 5. Top view SEM image of the sample $\mathrm{S} 2$ scale (a) $-5 \mu \mathrm{m}$ and (b) $-10 \mu \mathrm{m}$

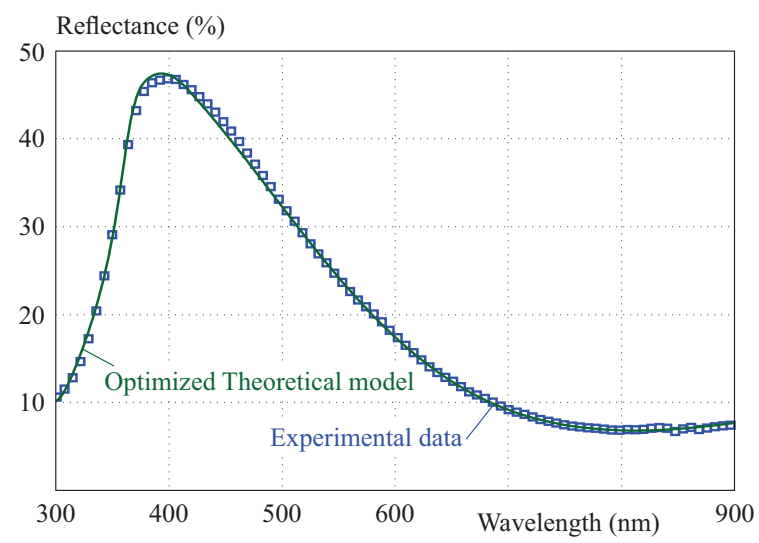

Fig. 6. Experimental data of $R$ of sample $\mathrm{S} 2$ and their optimized theoretical model

Table 2. Optimized parameters of Tauc-Lorentz dispersion model of sample $\mathrm{S} 1$, enegries in $\mathrm{eV}$

\begin{tabular}{lccccccc}
\hline$E_{g}$ & $\epsilon_{\infty}$ & $A_{1}$ & $A_{2}$ & $E_{1}$ & $E_{2}$ & $C_{1}$ & $C 2$ \\
2.97 & 4.39 & 88.62 & 109.96 & 3.48 & 6 & 1 & 0.1 \\
\hline
\end{tabular}

Electrochemical etching of n-type phosphor-doped $\mathrm{Si}$ substrate with resistivity $1-10 \Omega \mathrm{cm}$ (sample S2) caused the formation of a microstructure in contrast to sample $\mathrm{S} 1$, which has a porous structure. Formed microstructure exhibits diametrically different structural and optical properties than sample S1. The first significant difference compared to the sample $\mathrm{S} 1$ is the much greater homogeneity of the formed structure. The second difference lies in the $\mathrm{R}$ value of which is significantly higher. To de-

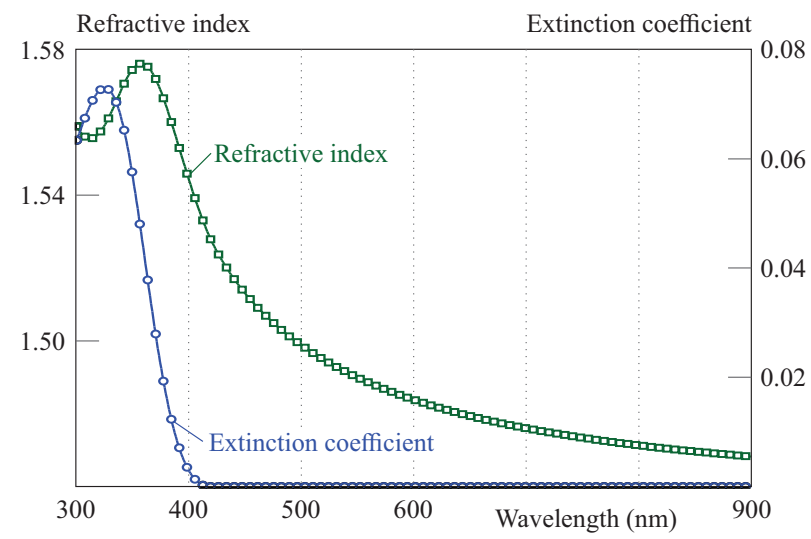

Fig. 7. Effective refractive index and extinction coefficient of sample S2

termine the effective thickness of the formed structure, theoretical models of $R$ and ellipsometric parameters of sample S2 were constructed and optimized. Theoretical model of $R$ of sample $\mathrm{S} 2$ was based on Looyenga effective medium approximation and Tauc-Lorentz dispersion model as in the case of sample S1. The construction of the theoretical model of $R$ and its optimization was less difficult than in the case of sample $\mathrm{S} 1$ due to the greater homogeneity of the structure. Construction and optimization of the theoretical model of the optical response of a homogeneous structure is generally simpler than in the case of inhomogeneous structures. The optimized parameters of Tauc-Lorentz dispersion model of sample S2 are listed in Tab. 3. Figure 6 shows the experimental data of $R$ of sample $\mathrm{S} 2$ and their optimized theoretical model. 


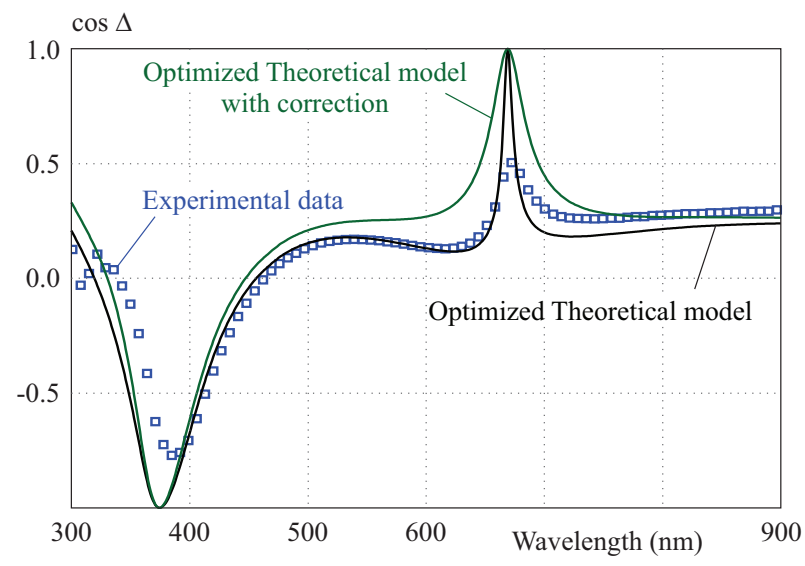

Fig. 8. Experimental data of $\cos \Delta$ of sample S2 and their optimized theoretical model

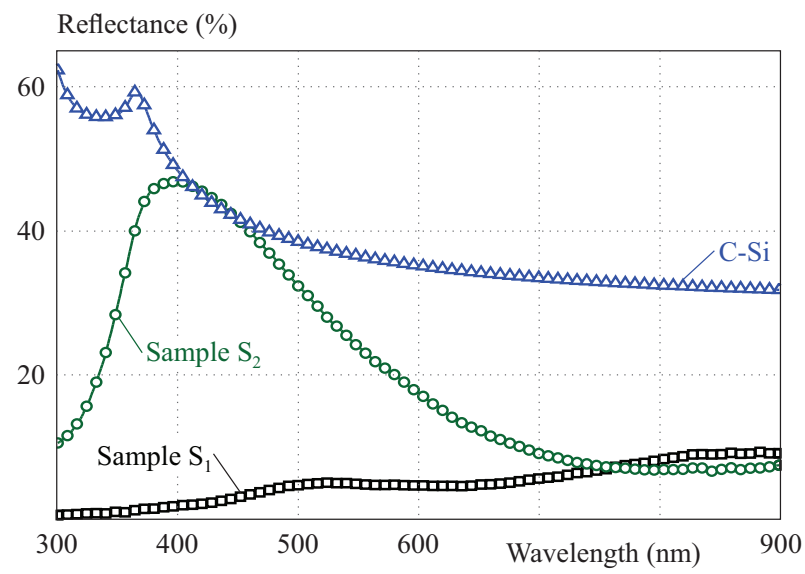

Fig. 10. Comparison of the reflectivity of samples S1, S2 and c-si

The effective refractive index and extinction coefficient of sample S2 obtained from optimized theoretical model of optical response are shown in Fig. 7. In addition to the optimization of the theoretical model of $R$, the optimization of the ellipsometric parameters $(\tan \Psi$ and $\cos \Delta)$ was also performed. The experimental data of ellipsometric parameters of sample S2 and their optimized theoretical model are shown in Fig. 8 and Fig. 9. The correction in the theoretical model of ellipsometric parameters was realized by including the roughness $(11 \mathrm{~nm})$ at the structure / substrate interface using modified Fresnel coefficients [23-24]

$$
\begin{aligned}
& t_{i, i-1}^{\mathrm{rgh}}=t_{i, i-1}^{\mathrm{o}} \exp \left[-\frac{1}{2}\left(\frac{2 \pi \sigma_{i}}{\lambda}\right)^{2}\left(n_{i-1}-n_{i}\right)^{2}\right], \\
& t_{i-1, i}^{\mathrm{rgh}}=t_{i-1, i}^{\mathrm{o}} \exp \left[-\frac{1}{2}\left(\frac{2 \pi \sigma_{i}}{\lambda}\right)^{2}\left(n_{i}-n_{i-1}\right)^{2}\right], \\
& r_{i, i-1}^{\mathrm{rgh}}=r_{i, i-1}^{\mathrm{o}} \exp \left[-2\left(\frac{2 \pi \sigma_{i} n_{i}}{\lambda}\right)^{2}\right], \\
& r_{i-1, i}^{\mathrm{rgh}}=r_{i-1, i}^{\mathrm{o}} \exp \left[-2\left(\frac{2 \pi \sigma_{i} n_{i-1}}{\lambda}\right)^{2}\right],
\end{aligned}
$$

where $r$ and $t$ with index ${ }^{\circ}$ are standard Fresnel coefficients, $\sigma_{i}$ is interface roughness $(\mathrm{nm}), n_{i}$ is refractive index of $i$-th layer and $\lambda$ is wavelength.

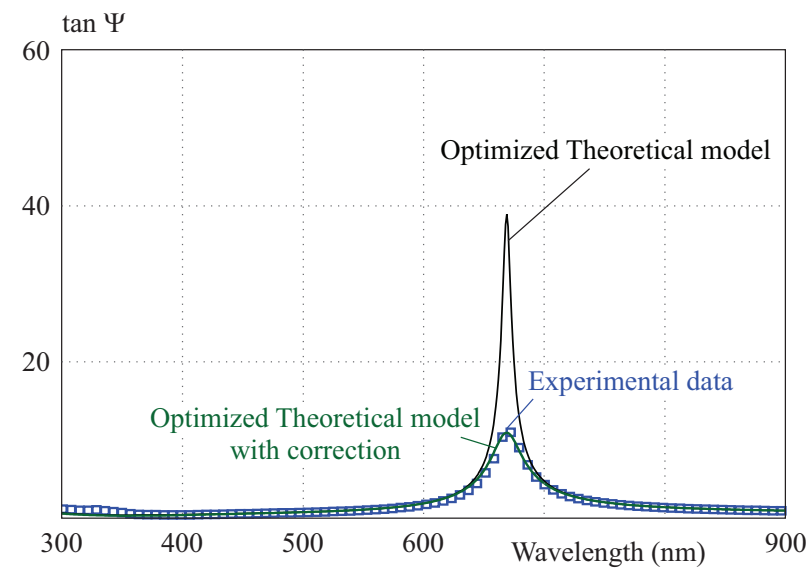

Fig. 9. Experimental data of $\tan \Psi$ of sample $S 2$ and their optimized theoretical model

Table 3. Optimized parameters of Tauc-Lorentz dispersion model of sample $\mathrm{S} 2$

\begin{tabular}{lccccccc}
\hline$E_{g}$ & $\epsilon_{\infty}$ & $A_{1}$ & $A_{2}$ & $E_{1}$ & $E_{2}$ & $C_{1}$ & $C_{2}$ \\
2.97 & 4.39 & 88.62 & 109.96 & 3.48 & 6 & 1 & 0.1 \\
\hline
\end{tabular}

The higher value of the reflectivity of the sample S2 compared to the sample S1 is with a high probability due to higher homogeneity of formed structure. Fig. 10 shows a comparison of the reflectivity of samples S1, S2 and crystalline silicon c-Si.

To compare the suppression of the reflectivity of prepared samples, the curves of experimental data of reflectivity were integrated. Value of area under the curve of reflectivity of crystalline silicon shown in Fig. 11(a) is 23415 , this value for the curve of reflectivity of sample S1 and S2 shown in Fig. 11(b,c) is 12242 and 2977. The decrease in reflectivity of sample $\mathrm{S} 2$ and $\mathrm{S} 1$ in the spectrum range from 300 to $900 \mathrm{~nm}$ compared to c-Si is $\sim 48 \%$ and $\sim 87 \%$.

\section{Conclusions}

This work dealt with the chemical surface treatment of silicon in order to reduce its surface reflectivity, as reflectivity has a significant influence on solar cell efficiency. Significant reduction of reflectivity was achieved for sample with high inhomogeneity of the formed porous structure (sample S1). The n-type phosphorus doped silicon wafers with different resistivity were used in the experiment. The surface treatment of this wafers was performed using the electrochemical etching method in HF-based solution. The silicon wafers were not excited by an external light source during the electrochemical etching process, except for the light source installed in the laboratory. Excitation by an external light source is necessary for the formation of homogenous porous structure in the case of use a n-type Si wafer. However, homogeneous porous structures do not achieve a sufficiently low reflectivity. 
Reflectance (\%)
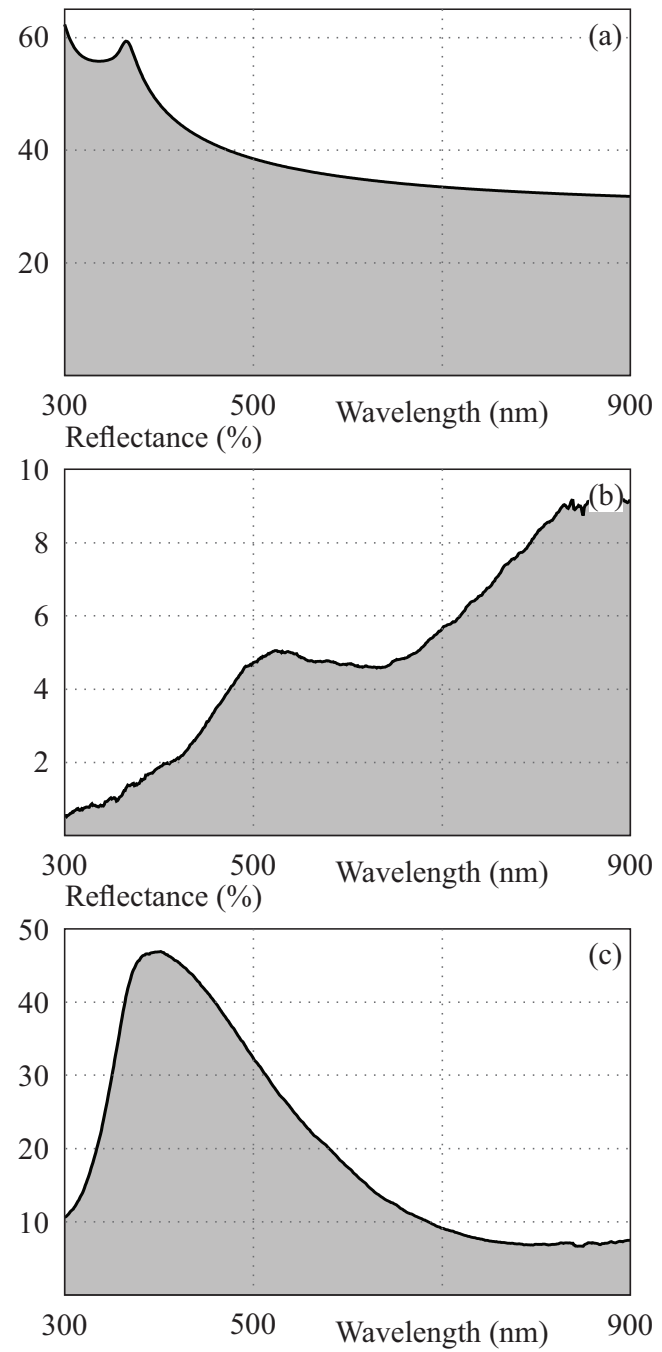

Fig. 11. Area under the curve of reflectivity of: (a) - crystalline silicon, (b) - sample S1, and (c) - sample S2

The formation of inhomogeneous porous structure with significantly suppressed reflectivity was observed when etching n-type silicon wafer with resistivity $\sim 0.06 \Omega \mathrm{cm}$ (sample S1). The decrease in reflectivity of sample S1 in the spectrum range from 300 to $900 \mathrm{~nm}$ compared to c-Si is $\sim 87 \%$. The effective pore diameter of sampl S1 is $\sim 188 \mathrm{~nm}$. The electrochemical etching of n-type silicon wafer with resistivity $1-10 \Omega \mathrm{cm}$ (sample S2) led to the formation of a more homogeneous microstructure with significantly higher value of reflectivity than sample S1. These experiments lead to the conclusion that the degree of homogeneity is significantly influenced by the type and concentration of dopants in the semiconductor.

\section{Acknowledgements}

The work was supported grant of Science and Technology Assistance Agency APVV-15-0152, Scientific Grant Agency of the Ministry of Education of Slovak Republic and the Slovak Academy of Sciences project VEGA $1 / 0676 / 17$.

\section{REFERENCES}

[1] K. WW. A. Chee, Z. Tang, H. Lü and F. Huang, "Anti-Reflective Structures for Photovoltaics: Numerical and Experimental Design", Energy Reports, vol. 4, pp. 266-273, 2018.

[2] G. Hashmi, M. J. Rashid, Z. H. Mahmood, M. Hoq and Md. H. Rahman, "Investigation of the Impact of Different ARC Layers using PC1D Simulation: Application to Crystalline Silicon Solar Cells", Journal of Theoretical and Applied Physics, vol. 12, pp. 327-334, 2018.

[3] H. Savin, P. Repo, G. von Gastrow, P. Ortega, E. Calle, M. Garín and R. Alcubilla, "Black Silicon Solar Cells with Interdigitated Back-Contacts Achieve 22.1\% Efficiency, Nature Nanotechnology, vol. 10, pp. 624-628, 2015.

[4] D. Losic and A. Santos, Electrochemically Engineered Nanoporous Materials Methods, Properties and Applications, Springer, ISBN 9783319203454, 2015.

[5] M. J. Sailor, Porous Silicon in Practice: Preparation, Characterization and Applications, Wiley-VCH, ISBN 9783527313785, 2012.

[6] V. Lehmann, Electrochemistry of Silicon: Instrumentation, Science, Materials and Applications, Wiley-VCH, ISBN 9783527293216 , 2002.

[7] S. Franssila, Inroduction to Microfabrication, Second edition, John Wiley \& Sons, ISBN 9780470749838, 2010.

[8] K. Imamura, Y. Onitsuka, Y. Sakae and H. Kobayashi, "High Conversion Efficiency of Crystalline Si Solar Cells using Black-Si Fabricated by SSCT Method", Journal of Electrical Engineering, vol. 68 , pp. 37-42, 2017.

[9] E. E. Antunez, U. Salazar-Kuri, J. O. Estevez, J. Campos, M. A. Basurto, S. Jiménez Sandoval and V. Agarwal, "Porous Silicon-VO2 Based Hybrids as Possible Optical Temperature Sensor: Wavelengthdependent", Journal of Applied Physics, vol. 118 , no. 13 , pp. 1-8, 2015.

[10] B. Fodor, E. Agocs, B. Bardet, T. Defforge, F. Cayrel, D. Alquier, M. Fried, G. Gautier and P. Petrik, "Porosity and Thickness Characterization of Porous Si and Oxidized Porous Si Layers an Ultraviolet-Visible-Mid Infrared Ellipsometry Study", Microporous and Mesoporous Materials, vol. 227, pp. 112-120, 2016.

[11] X. Liu, P. R. Coxon, M. Peters, B. Hoex, J. M. Cole and D. J. Fray, "Black Silicon Fabrication Methods, Properties and Solar Energy Applications", Energy \& Environmental Science, vol. 7, no. 10 , pp. $3223-3263,2014$.

[12] J. Sakai, E. Luais, J. Wolfman, T. Tillocher, R. Dussart, F. Tran-Van and F. Ghamouss, "Cryogenic Plasma-Processed Silicon Microspikes as a High-Performance Anode Material for Lithium Ion-Batteries", Journal of Applied Physics, vol. 122, no. 15 , pp. $1-7,2017$.

[13] Z. Xu, Y. Yao, E. P. Brueckner, L. Li, J. Jiang, R. G. Nuzzo and G. L. Liu, "Black Silicon Solar Thin-Film Microcells Integrating Top Nanocone Structures for Broadband and Omnidirectional Light-Trapping", Nanotechnology, vol. 25, no. 30, pp. 1-8, 2014.

[14] F. A. Harraz, " Porous Silicon Chemical Sensors and Biosensors: A Review", Sensors and Actuators B: Chemical, vol. 202, pp. 897-912, 2014.

[15] R. J. Martín-Palma, M. Manso-Silván and V. Torres-Costa, "Biomedical Applications of Nanostructured Porous Silicon: a Review", Journal of Nanophotonics, vol. 4, pp. 1-20, 2010.

[16] A. Jane, R. Dronov, A. Hodges and N. H. Voelcker, "Porous Silicon Biosensors on the Advance", Trends in Biotechnology, vol. 27, no. 4, pp. 230-239 2009.

17] M. Kroll, M. Otto, T. Käsebier, K. Füchsel, R. Wehrspohn, E.-B. Kley, A. Tünnermann and T. Pertsch, "Black Silicon for Solar Cell Applications", Photonics for Solar Energy Systems IV, vol. 843817, pp. 1-11, 2012. 
[18] M. Otto, M. Algasinger, H. Branz, B. Gesemann, T. Gimpel, K. Füchsel, T. Käsebier, S. Kontermann, S. Koynov, X. Li, V. Naumann, J. Oh, A. N. Sprafke, J. Ziegler, M. Zilk and R. B. Wehrspohn, "Black Silicon Photovoltaics", Advanced Optical Materials, vol. 3, no. 2, pp. 147-164, 2014.

[19] V. Torres-Costa, F. Agulló-Rueda, R. J. Martín-Palma and J. M. Martínez-Duart, "Porous Silicon Optical Devices for Sensing Applications", Optical Materials, vol. 27, no. 5, pp. 1084-1087, 2004.

[20] J. Sakabe, N. Ohta, T. Ohnishi, K. Mitsuishi and K. Takada, "Porous Amorphous Silicon Film Anodes for High Capacity and Stable All-Solid-State Lithium Batteries", Communications Chemistry, vol. 1, no. 24, pp. 1-9, 2018.

[21] C. F. Ramirez-Gutierrez, J. D. Castaño-Yepes and M. E. Rodriguez-Garcia, "Porosity and Roughness Determination of
Porous Silicon Thin Films by Genetic Algorithms", Optik, vol. 173, pp. 271-278, 2018.

22] G. E. Jellison and F. A. Modine, "Parameterization of the Optical Functions of Amorphous Materials in the Interband Region", Applied Physics Letters, vol. 69, no. 3, pp. 371-373, 1996.

23] C. C. Katsidis and D. I. Siapkas, "General Transfer-Matrix Method for Optical Multilayer Systems with Coherent, Partially Coherent, and Incoherent Interference", Applied Optics, vol. 41, no. 19, pp. 3978-3987, 2002.

[24] C. L. Mitsas and D. I. Siapkas, "Generalized Matrix Method for Analysis of Coherent and Incoherent Reflectance and Transmittance of Multilayer Structures with Rough Surfaces, Interfaces, and Finite Substrates", Applied Optics, vol. 34, no. 10, pp. 1678-1683, 1995.

Received 2 July 2020 